\title{
Imagem de uma cônica segundo uma transformação linear no no plano
}

\author{
Image of a cone according to a linear transformation of the plane
}

\author{
Fabiano Pinto Tavares e Uilbiran Chaves Santos \\ Instituto Federal do Maranhão,MA, Brasil \\ fabiano.tavares@ifma.edu.br; uilchaves28@ifma.edu.br
}

\begin{abstract}
Resumo
Analisa-se, neste trabalho, as deformações de cônicas por transformações lineares. Usa-se a forma paramétrica de uma cônica para encontrar a equação de sua imagem quando o operador linear for associado a uma matriz de posto incompleto. No caso em que o operador linear é invertível, estuda-se os autovalores da matriz simétrica associada à forma quadrática da cônica original e a partir daí encontra-se a equação de sua imagem no referencial gerado pelos autovetores desta matriz.
\end{abstract}

Palavras-chave: Autovalores, Cônicas, Transformações lineares.

\begin{abstract}
It is analyzed in this work, the conical deformation by linear transformations. It uses the parametric form of a conic to find the equation of your image when the linear operator is associated with an incomplete set of matrix. In the event that the linear operator is invertible, we study the eigenvalues of the symmetric matrix associated with the quadratic form of the original cone and from there is the equation of your image in the frame generated by the eigenvectors of this matrix.
\end{abstract}

Keywords: Eigenvalues, Conical, linear transformations. 


\section{Introdução}

Esta seção do trabalho tem por objetivo Introduzir os conceitos de autovalores e autovetores de um operador linear bem como de uma matriz.

De acordo com Lima (1996), se $V$ é um espaço vetorial e $T$ um operador linear definido sobre $V$, encontra-se vetores em $V$ que são levados em seus múltiplos escalares segundo o operador $T$, de acordo com a definição 1 .

Definição 1. Seja $V$ um espaço vetorial e seja $T: V \rightarrow V$ um operador linear. Caso existam $v \in V, v \neq 0 e \lambda \in \mathbb{R}$ tal que $T(v)=\lambda v$, diz-se que $\lambda$ é um autovalor de $T$ associado ao autovetor $v$ de $T$.

Ao considerar operadores definidos no plano (ou no espaço), isto é, $V=\mathbb{R}^{2}$ (ou $V=\mathbb{R}^{3}$ ) tem-se que a imagem de um autovetor $v$, caso exista, estará sobre a mesma reta que contêm $v$. O autovalor $\lambda$ por sua vez determina o sentido e o comprimento de $T(v)$.

De acordo com Howard e Rorres (2001), para determinar autovalores e autovetores de um operador linear $T: V \rightarrow V$, fixando uma base $\alpha$ para $V$, pode-se reescrever a equação $T(v)=\lambda v$ na forma matricial $A(v)=\lambda v$, onde $A$ é a matriz do operador $T$ na base $\alpha$, ou seja, $A=[T]_{\alpha}^{\alpha}$ e o vetor $v$ deverá ser considerado um vetor coluna. Desta forma, tem-se:

$$
A(v)=\lambda v \Leftrightarrow(A-\lambda I) v=0 .
$$

A última equação representa um sistema linear homogêneo e para que exista $v \neq 0$ que o satisfaça é necessário e suficiente que $\operatorname{det}(A-\lambda I)=0$. Logo, os autovalores de $T$ serão as raízes do polinômio

$$
P(\lambda)=\operatorname{det}(A-\lambda I) .
$$

De acordo com Boldrini et al. (1986), um fato importante é que este polinômio não depende da base fixada para o espaço $V$. Com efeito, o estudo das transformações lineares e matrizes garante que $B=C \cdot A \cdot C^{-1}$, onde B é a matriz do operador T em uma base $\beta$ arbitrária, ou seja, $B=[T]_{\beta}^{\beta}$, e $C$ é a matriz de mudança da base $\alpha$ para a base $\beta$, ou seja, $C=[I]_{\beta}^{\alpha}$. Logo, tem-se:

$$
\begin{aligned}
\operatorname{det}(B-\lambda I) & =\operatorname{det}\left(C \cdot A \cdot C^{-1}-\lambda I\right) \\
& =\operatorname{det}\left(C \cdot A \cdot C^{-1}-\lambda C \cdot I \cdot C^{-1}\right) \\
& =\operatorname{det}\left(C(A-\lambda I) C^{-1}\right) \\
& =\operatorname{det}(C) \operatorname{det}(A-\lambda I) \operatorname{det}\left(C^{-1}\right) \\
& =\operatorname{det}(A-\lambda I)=P(\lambda) .
\end{aligned}
$$

Este polinômio $P(\lambda)$ é chamado polinômio característico do operador $T$ e suas raízes são os autovalores de T. Segundo Coelho e Lourenço (2001), para cada $\lambda$ resolve-se o sistema indeterminado $(A-\lambda I) v=0$ para se determinar os autovetores associados. Na verdade, da solução do sistema $\left(A-\lambda_{1} I\right) v=0$, segue que todo múltiplo escalar de $v$ é um autovetor associado a $\lambda$. Daqui por diante fica subtendido que quando se diz que $v$ é um autovetor associado ao autovalor $\lambda$ segundo um operador $T$, então todo múltiplo escalar de $v$ também o será.

Por exemplo, considere a matriz simétrica

$$
A=\left(\begin{array}{ll}
a & b \\
b & c
\end{array}\right) .
$$

O polinômio característico de $A$ é

$$
P(\lambda)=\operatorname{det}(A-\lambda I)=\lambda^{2}-(a+c) \lambda+\left(a c-b^{2}\right) .
$$

O discriminante de $P(\lambda)=0$ é $\Delta=(a-c)^{2}+4 b^{2}$. Como $\Delta \geq 0$ segue que $A$ possui dois autovalores, os quais são dados por

$$
\lambda=\frac{(a+c) \pm \sqrt{(a-c)^{2}+4 b^{2}}}{2} .
$$

De acordo com Lang (2004), para encontrar os autovetores associados resolve-se o sistema indeterminado $\left(A-\lambda_{i} I\right) v=0$, o qual é representado em forma matricial como:

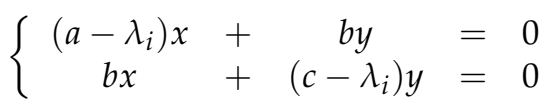

Aplicando a operação elementar

$$
E:-b L_{1}+\left(a-\lambda_{i}\right) L_{2} \rightarrow L_{2}
$$

no sistema acima obtêm-se que

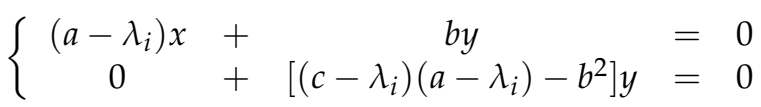

Note que, para se realizar a operação elementar $E$, deve-se ter $\lambda_{i} \neq a$. Como $\lambda_{i}$ é autovalor de $A$ tem-se que

$$
\left(c-\lambda_{i}\right)\left(a-\lambda_{i}\right)-b^{2}=0,
$$

logo encontra-se como solução do sistema $x=\frac{b}{\lambda_{i}-a} y$. Portanto, se $\lambda_{i} \neq a$ tem-se que o autovetor associado ao autovalor $\lambda_{i}$ será $\left(\frac{b}{\lambda_{i}-a}, 1\right), i=1,2$. Neste caso, os vetores

$$
\left(\frac{b}{\lambda_{1}-a}, 1\right) \text { e }\left(\frac{b}{\lambda_{2}-a}, 1\right)
$$

são ortogonais. Com efeito,

$$
\begin{aligned}
& \left\langle\left(\frac{b}{\lambda_{1}-a}, 1\right),\left(\frac{b}{\lambda_{2}-a}, 1\right)\right\rangle= \\
= & \frac{b^{2}}{\left(\lambda_{1}-a\right)\left(\lambda_{2}-a\right)}+1 \\
= & \frac{b^{2}}{\lambda_{1} \lambda_{2}-\left(\lambda_{1}+\lambda_{2}\right) a+a^{2}}+1
\end{aligned}
$$




$$
\begin{aligned}
& =\frac{b^{2}}{\left(a c-b^{2}\right)-(a+c) a+a^{2}}+1 \\
& =0 .
\end{aligned}
$$

Se $\lambda_{i}=a$ então $b=0$. De fato,

$$
\begin{aligned}
\lambda_{i}=\frac{(a+c) \pm \sqrt{(a-c)^{2}+4 b^{2}}}{2} & =a \\
\pm \sqrt{(a-c)^{2}+4 b^{2}} & =a-c \\
(a-c)^{2}+4 b^{2} & =(a-c)^{2} \\
b & =0 .
\end{aligned}
$$

Portanto, se $\lambda_{i}=a$ tem-se duas situações:

$\left.1^{\underline{a}}\right) a \neq c$. Aqui, a matriz $A$ é diagonal, então tem-se o autovetor $(1,0)$ associado ao autovalor $a$ e o autovetor $(0,1)$ associado ao autovalor $c$.

$\left.2^{a}\right) \quad a=c$. Aqui, a matriz $A$ é diagonal e múltipla da identidade, então todo vetor não nulo do $\mathbb{R}^{2}$ é autovetor associado ao autovalor $a=c$. Desta forma, acaba-se de mostrar a proposição 1 .

Proposição 1. Seja

$$
A=\left(\begin{array}{ll}
a & b \\
b & c
\end{array}\right)
$$

simétrica. Seus autovalores são dados por:

$$
\lambda=\frac{(a+c) \pm \sqrt{(a-c)^{2}+4 b^{2}}}{2} .
$$

Tem-se também que:

(i) Se $\lambda_{i} \neq a$, então o autovetor associado ao autovalor $\lambda_{i}$ será

$$
\left(\frac{b}{\lambda_{i}-a}, 1\right), i=1,2
$$

(ii) Se $\lambda_{i}=a$, tem-se:

$1^{o}$ caso: $a \neq c$. Neste caso, $(1,0)$ é o autovetor associado ao autovalor a e $(0,1)$ é o autovetor associado ao autovalor $c$.

$2^{-}$caso: $a=c$. Neste caso, todo vetor não nulo do $\mathbb{R}^{2}$ é autovetor associado ao autovalor $a=c$.

Os conceitos apresentados nesta seção são importantes, pois serão utilizados para estudar a deformação de uma cônica por transformações lineares. No caso em que o operador linear é invertível, estuda-se os autovalores da matriz simétrica associada à forma quadrática da cônica original e a partir daí encontra-se a equação de sua imagem no referencial gerado pelos autovetores desta matriz.

\section{Deformação da elipse}

Nesta seção, analisa-se a imagem de uma elipse segundo um operador linear no plano. Seja $T_{M}: \mathbb{R}^{2} \rightarrow \mathbb{R}^{2}$ um operador linear definido por:

$$
T_{M}\left(\begin{array}{l}
x \\
y
\end{array}\right)=M\left(\begin{array}{l}
x \\
y
\end{array}\right)=\left(\begin{array}{ll}
a & b \\
c & d
\end{array}\right)\left(\begin{array}{l}
x \\
y
\end{array}\right)
$$

e uma elipse qualquer (que pode estar rotacionada e transladada em relação ao referencial $x O y$ ), mostra-se que a imagem da elipse segundo $T_{M}$ será uma elipse ou suas degenerações (ponto ou segmento de reta). Nas subseções 2.1 a 2.4 estuda-se uma elipse $\epsilon$ de equação

$$
\frac{x^{2}}{p^{2}}+\frac{y^{2}}{q^{2}}=1
$$

onde serão discutidos todos os possíveis tipos de operadores no plano.

\section{1 $\epsilon$ é uma elipse centrada na origem e não rotacionada e $T_{M}$ é o operador nulo}

Neste caso, a imagem da elipse será o vetor nulo, já que $T_{M}(v)=0$ para todo $v \in \mathbb{R}^{2}$. Desta forma, a imagem da elipse é um ponto (elipse degenerada).

\section{$2.2 \epsilon$ é uma elipse centrada na origem e não rotacionada e $T_{M}$ é um operador não nulo onde $\operatorname{posto}(M)=1$}

Neste caso, tem-se $a d-b c=0, \log$ os vetores $\left(\begin{array}{l}a \\ b\end{array}\right)$ e $\left(\begin{array}{l}c \\ d\end{array}\right)$ são linearmente dependente. Supõe-se, sem perda de generalidade, que

$$
\left(\begin{array}{l}
c \\
d
\end{array}\right)=k\left(\begin{array}{l}
a \\
b
\end{array}\right) \text {. }
$$

Pode-se então escrever

$$
T_{M}\left(\begin{array}{l}
x \\
y
\end{array}\right)=\left(\begin{array}{cc}
a & b \\
k a & k b
\end{array}\right)\left(\begin{array}{l}
x \\
y
\end{array}\right) .
$$

Tomando a representação paramétrica da elipse temse

$$
\left(\begin{array}{l}
x \\
y
\end{array}\right)=\left(\begin{array}{c}
p \cos (t) \\
q \sin (t)
\end{array}\right), t \in[0,2 \pi]
$$

$\log 0$

$$
\begin{aligned}
T_{M}\left(\begin{array}{l}
x \\
y
\end{array}\right) & =T_{M}\left(\begin{array}{c}
p \cos (t) \\
q \sin (t)
\end{array}\right) \\
& =\left(\begin{array}{cc}
a & b \\
k a & k b
\end{array}\right)\left(\begin{array}{l}
p \cos (t) \\
q \sin (t)
\end{array}\right)
\end{aligned}
$$




$$
\begin{aligned}
& =(a p \cos (t)+b q \sin (t))\left(\begin{array}{l}
1 \\
k
\end{array}\right) \\
& =\bar{x}\left(\begin{array}{l}
1 \\
k
\end{array}\right)
\end{aligned}
$$

onde $\bar{x}=a p \cos (t)+b q \sin (t)$. Já que $\bar{x}$ é limitada,

$$
\bar{x}\left(\begin{array}{l}
1 \\
k
\end{array}\right)
$$

representa a equação paramétrica de um segmento da reta cujo vetor diretor é $\left(\begin{array}{l}1 \\ k\end{array}\right)$.

Desta forma, a imagem da elipse é um segmento de reta (elipse degenerada). Determina-se tal segmento de reta. O máximo e o mínimo de $\bar{x}$ nos permitirão encontrar as extremidades do segmento. Derivando $\bar{x}$ tem-se

$$
\frac{d \bar{x}}{d t}=b q \cos (t)-a p \sin (t) .
$$

Resolvendo a equação $\frac{d \bar{x}}{d t}=0$ tem-se $\tan (t)=\frac{b q}{a p}$ caso $a \neq 0$ (se $a=0$ então $b \neq 0$ daí teria-se $t=\frac{\pi}{2}$ ).

1ํㅡㄴ $a b>0$

Supondo que $a>0$ e $b>0$ (se $a<0$ e $b<0$ seria análogo) tem-se $\tan (t)=\frac{b q}{a p}>0$. Neste caso, a equação tem soluções para $t$ no $1^{\underline{o}}$ e $3^{o}$ quadrantes. Logo, tem-se $t_{1}=\arctan \left(\frac{b q}{a p}\right)$ e $t_{2}=\pi+\arctan \left(\frac{b q}{a p}\right)$ que são arcos do $1^{\underline{o}}$ e $3^{o}$ quadrantes respectivamente (aqui considera-se que a função arco tangente está definida no intervalo $\left(-\frac{\pi}{2}, \frac{\pi}{2}\right)$ ). Encontrando a derivada segunda de $\bar{x}$ tem-se

$$
\frac{d^{2} \bar{x}}{d t^{2}}=-(b q \sin (t)+a p \cos (t))
$$

Como $t_{1}$ está no $1^{o}$ quadrante, $a>0$ e $b>0$ tem-se $\frac{d^{2} \bar{x}}{d t^{2}}\left(t_{1}\right)<0$. Logo, $t_{1}$ é ponto de máximo. Como $t_{2}$ está no $3^{\circ}$ quadrante, $a>0$ e $b>0$ tem-se

$$
\frac{d^{2} \bar{x}}{d t^{2}}\left(t_{2}\right)>0
$$

Segue que $t_{2}$ é ponto de mínimo. Logo, o segmento de reta tem extremidades nos pontos

$$
P_{1}=\left(a p \cos \left(t_{1}\right)+b q \sin \left(t_{1}\right), k a p \cos \left(t_{1}\right)+k b q \sin \left(t_{1}\right)\right)
$$

e

$$
P_{2}=\left(a p \cos \left(t_{2}\right)+b q \sin \left(t_{2}\right), k a p \cos \left(t_{2}\right)+k b q \sin \left(t_{2}\right)\right)
$$

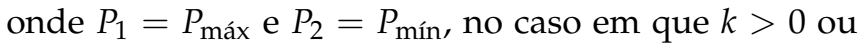
$P_{1}=P_{\text {mín }}$ e $P_{2}=P_{\text {máx }}$, no caso em que $k<0$.

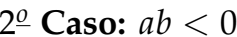

Supondo que $a>0$ e $b<0$ (se $a<0$ e $b>0$ seria análogo) tem-se $\tan (t)=\frac{b q}{a p}<0$. Neste caso, a equação tem soluções para $t$ no $2^{o}$ e $4^{o}$ quadrantes. Logo, tem-se

$$
t_{1}=\arctan \left(\frac{b q}{a p}\right) \text { e } t_{2}=\pi+\arctan \left(\frac{b q}{a p}\right)
$$

que são arcos do $2^{\circ}$ e $4^{\circ}$ quadrantes, respectivamente. Aqui, considera-se que a função arco tangente está definida no intervalo $\left(\frac{\pi}{2}, \frac{3 \pi}{2}\right)$. Encontrando a derivada segunda de $\bar{x}$ tem-se

$$
\frac{d^{2} \bar{x}}{d t^{2}}=-(b q \sin (t)+a p \cos (t))
$$

Como $t_{1}$ está no $2^{o}$ quadrante, $a>0$ e $b<0$ te-se $\frac{d^{2} \bar{x}}{d t^{2}}\left(t_{1}\right)>0$. Logo, $t_{1}$ é ponto de mínimo. Como $t_{2}$ está no $4^{o}$ quadrante, $a>0$ e $b<0$ tem-se $\frac{d^{2} \bar{x}}{d t^{2}}\left(t_{2}\right)<0$. Segue que $t_{2}$ é ponto de máximo. Logo, o segmento de reta tem extremidades nos pontos

$$
P_{1}=\left(a p \cos \left(t_{1}\right)+b q \sin \left(t_{1}\right), k a p \cos \left(t_{1}\right)+k b q \sin \left(t_{1}\right)\right)
$$

$\mathrm{e}$

$$
P_{2}=\left(a p \cos \left(t_{2}\right)+b q \sin \left(t_{2}\right), k a p \cos \left(t_{2}\right)+k b q \sin \left(t_{2}\right)\right)
$$

onde $P_{1}=P_{\text {mín }}$ e $P_{2}=P_{\text {máx }}$, no caso em que $k>0$ ou $P_{1}=P_{\text {máx }}$ e $P_{2}=P_{\text {mín }}$, no caso em que $k<0$.

\section{$3^{-}$Caso: $a b=0$}

Neste caso, $b=0$ já que $a \neq 0$. Assim, tem-se $\bar{x}=a p \cos (t)$. Supondo $a>0$ (se $a<0$ seria análogo) tem-se $t_{1}=t_{\text {máx }}=0$ e $t_{2}=t_{\text {mín }}=\pi$. Logo, o segmento de reta têm extremidades nos pontos

$$
P_{1}=(a p, k a p) \text { e } P_{2}=(-a p,-k a p)
$$

onde $P_{1}=P_{\text {máx }}$ e $P_{2}=P_{\text {mín, no caso em que } k>0 \text { ou }}$ $P_{1}=P_{\text {mín }}$ e $P_{2}=P_{\text {máx }}$, no caso em que $k<0$.

Em qualquer caso os extremos do segmento dados pelos pontos $P_{1}$ e $P_{2}$ são simétricos em relação a origem e ainda o comprimento deste é dado por

$$
\begin{array}{r}
\left\|\overline{P_{1} P_{2}}\right\|=\sqrt{\left(1+k^{2}\right)} \mid a p\left[\cos \left(t_{2}\right)-\cos \left(t_{1}\right)\right] \\
+b q\left[\sin \left(t_{2}\right)-\sin \left(t_{1}\right)\right] \mid
\end{array}
$$

Com isso, tem-se a proposição 2. 
Proposição 2. Seja M uma matriz $2 \times 2$ de posto 1 , ou seja, $M$ é de uma das formas:

$$
\begin{gathered}
\left(\begin{array}{cc}
a & b \\
k a & k b
\end{array}\right) \text { ou }\left(\begin{array}{cc}
k a & k b \\
a & b
\end{array}\right), \\
(a, b) \neq(0,0) .
\end{gathered}
$$

Seja $T_{M}: \mathbb{R}^{2} \rightarrow \mathbb{R}^{2}$ um operador associado a matriz $M$ na base canônica. Se є é uma elipse de equação

$$
\frac{x^{2}}{p^{2}}+\frac{y^{2}}{q^{2}}=1
$$

então $T_{M}(\epsilon)$ é o segmento $\overline{P_{1} P_{2}}$ da reta cujo vetor diretor é $(1, k)$ ou $(k, 1)$ conforme $M$ tenha respectivamente a $1^{\underline{a}}$ ou $2^{-}$ representação dada anteriormente, onde $P_{1}$ e $P_{2}$ são simétricos em relação a origem. Este segmento tem comprimento dado pela fórmula (2) sendo

$$
t_{1}=\arctan \left(\frac{b q}{a p}\right), t_{2}=\pi+\arctan \left(\frac{b q}{a p}\right)(\operatorname{casoa} \neq 0)
$$

ou

$$
t_{1}=\frac{\pi}{2}, \quad t_{2}=\frac{3 \pi}{2}, \operatorname{caso}(a=0)
$$

\section{3 $\epsilon$ é uma elipse centrada na origem e não rotacionada e $T_{M}$ é um operador invertí- vel}

Mostra-se que a imagem de $\epsilon$ segundo o operador $T_{M}$ é uma elipse cuja equação determina-se. Reescrevendo a equação de $\epsilon$ em forma matricial tem-se:

$$
\left(\begin{array}{ll}
x & y
\end{array}\right)\left(\begin{array}{cc}
\frac{1}{p^{2}} & 0 \\
0 & \frac{1}{q^{2}}
\end{array}\right)\left(\begin{array}{l}
x \\
y
\end{array}\right)=1
$$

Admitindo que $T_{M}(x, y)=(u, v)$ e que $T_{M}$ é invertível tem-se que

$$
\left(\begin{array}{l}
x \\
y
\end{array}\right)=M^{-1}\left(\begin{array}{l}
u \\
v
\end{array}\right)
$$

Substituindo este resultado em (3) obtém-se

$$
\begin{array}{r}
\left(\begin{array}{ll}
u & v
\end{array}\right)\left(M^{-1}\right)^{t}\left(\begin{array}{cc}
\frac{1}{p^{2}} & 0 \\
0 & \frac{1}{q^{2}}
\end{array}\right) M^{-1}\left(\begin{array}{l}
u \\
v
\end{array}\right)=1 \\
\left(\begin{array}{ll}
u & v
\end{array}\right) S\left(\begin{array}{l}
u \\
v
\end{array}\right)=1,
\end{array}
$$

onde

$$
S=\left(M^{-1}\right)^{t}\left(\begin{array}{cc}
\frac{1}{p^{2}} & 0 \\
0 & \frac{1}{q^{2}}
\end{array}\right) M^{-1} .
$$

Note que $S$ é simétrica, então existe $P$ ortonormal e $D$ diagonal tal que $S=P D P^{t}$. Desta forma, (4) pode ser assim reescrita

$$
\begin{array}{r}
\left(\begin{array}{ll}
u & v
\end{array}\right) P\left(\begin{array}{cc}
\lambda_{1} & 0 \\
0 & \lambda_{2}
\end{array}\right) P^{t}\left(\begin{array}{l}
u \\
v
\end{array}\right)=1 \\
\left(\begin{array}{cc}
\widetilde{u} & \widetilde{v}
\end{array}\right)\left(\begin{array}{cc}
\lambda_{1} & 0 \\
0 & \lambda_{2}
\end{array}\right)\left(\begin{array}{c}
\widetilde{u} \\
\widetilde{v}
\end{array}\right)=1 \\
\lambda_{1}(\widetilde{u})^{2}+\lambda_{2}(\widetilde{v})^{2}=1,
\end{array}
$$

onde

$$
\left(\begin{array}{l}
u \\
v
\end{array}\right)=P\left(\begin{array}{c}
\widetilde{u} \\
\widetilde{v}
\end{array}\right)
$$

$\mathrm{e}$

$$
D=\left(\begin{array}{cc}
\lambda_{1} & 0 \\
0 & \lambda_{2}
\end{array}\right)
$$

A matriz $S$ é dada por

$$
S=\left(\begin{array}{ll}
\widetilde{a} & \widetilde{b} \\
\widetilde{b} & \widetilde{c}
\end{array}\right)
$$

onde

$$
\begin{gathered}
\widetilde{a}=\frac{d^{2} q^{2}+c^{2} p^{2}}{(\operatorname{det} M)^{2} p^{2} q^{2}} \\
\widetilde{b}=\frac{-b d q^{2}-a c p^{2}}{(\operatorname{det} M)^{2} p^{2} q^{2}} \\
\widetilde{c}=\frac{b^{2} q^{2}+a^{2} p^{2}}{(\operatorname{det} M)^{2} p^{2} q^{2}}
\end{gathered}
$$

Um dos autovalores de $S$ será

$$
\lambda_{1}=\frac{(\widetilde{a}+\widetilde{c})+\sqrt{(\widetilde{a}-\widetilde{c})^{2}+4 \widetilde{b}^{2}}}{2} .
$$

Mas, $\widetilde{a}+\widetilde{c}>0$. Logo, $\lambda_{1}>0$. O determinante de $S$ é

$$
\frac{1}{(\operatorname{det} M)^{2} p^{2} q^{2}}>0,
$$

daí segue que $S$ têm os dois autovalores positivos, o que acarreta no fato da imagem de $\epsilon$ segundo $T_{M}$, que é dada pela equação (5), ser uma elipse. Com isso, acabase de demonstrar a proposição que se segue.

Proposição 3. Seja $T_{M}: \mathbb{R}^{2} \rightarrow \mathbb{R}^{2}$ um operador linear invertível associado a matriz $M$ na base canônica. Se є é uma elipse de equação

$$
\frac{x^{2}}{p^{2}}+\frac{y^{2}}{q^{2}}=1
$$

então $T_{M}(\epsilon)$ é uma elipse dada pela equação (5), onde $\lambda_{1}$ e $\lambda_{2}$ são os autovalores da matriz

$$
S=\left(M^{-1}\right)^{t}\left(\begin{array}{cc}
\frac{1}{p^{2}} & 0 \\
0 & \frac{1}{q^{2}}
\end{array}\right) M^{-1}
$$


e $\widetilde{u} \widetilde{v}$ é o referencial gerado pelos respectivos autovetores ortonormais desta matriz.

Exemplo 1. Considere a elipse

$$
\frac{x^{2}}{4}+\frac{y^{2}}{9}=1
$$

segundo a transformação

$$
T_{M}\left(\begin{array}{l}
x \\
y
\end{array}\right)=\left(\begin{array}{ll}
1 & 1 \\
2 & 2
\end{array}\right)\left(\begin{array}{l}
x \\
y
\end{array}\right) .
$$

Para isso, escreve-se a elipse parametricamente

$$
f(t)=\left(\begin{array}{c}
2 \cos (t) \\
3 \sin (t)
\end{array}\right)
$$

onde $t \in[0,2 \pi]$.

Note que, neste caso, a imagem da elipse é um segmento de reta $\overline{P_{1} P_{2}}$ cujo vetor diretor é $(1,2)$, pois $\operatorname{det} M=0$. $O$ comprimento deste segmento é dado por $\frac{26 \sqrt{5}}{\sqrt{13}}$. O gráfico apresentado na figura 1 e os demais gráficos desse documento, foram construídos utilizando-se do aplicativo computacional GeoGebra.

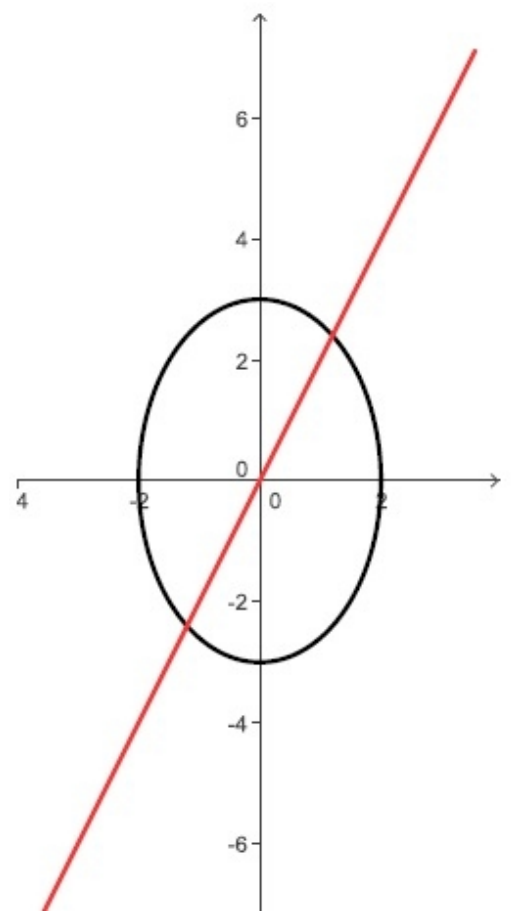

Figura 1: Considere a elipse de equação $\frac{x^{2}}{4}+\frac{y^{2}}{9}=1$ segundo o operador $T(x, y)=(x+y, 2 x+2 y)$.

Exemplo 2. Veja a imagem da elipse $\frac{x^{2}}{4}+\frac{y^{2}}{9}=1$ segundo a transformação

$$
T_{M}\left(\begin{array}{l}
x \\
y
\end{array}\right)=\left(\begin{array}{ll}
1 & 2 \\
3 & 4
\end{array}\right)\left(\begin{array}{l}
x \\
y
\end{array}\right) .
$$

Note que como det $M \neq 0$, a imagem da elipse é uma elipse de equação

$$
\left(\frac{55-7 \sqrt{61}}{72}\right)(\widetilde{u})^{2}+\left(\frac{7 \sqrt{61}+55}{72}\right)(\widetilde{v})^{2}=1,
$$

onde $\widetilde{u} \widetilde{v}$ é o referencial gerado pela base

$$
\begin{aligned}
& \left\{\left(\frac{3 \sqrt{2}}{\sqrt{5 \sqrt{61}+61}}, \frac{\sqrt{2}(\sqrt{61}+5)}{2 \sqrt{5 \sqrt{61}+61}}\right),\right. \\
& \left.\left(\frac{3 \sqrt{2}}{\sqrt{61-5 \sqrt{61}}}, \frac{\sqrt{2}(5-\sqrt{61})}{2 \sqrt{61-5 \sqrt{61}}}\right)\right\} .
\end{aligned}
$$

O gráfico de tal elipse pode ser visualizado na figura 2.

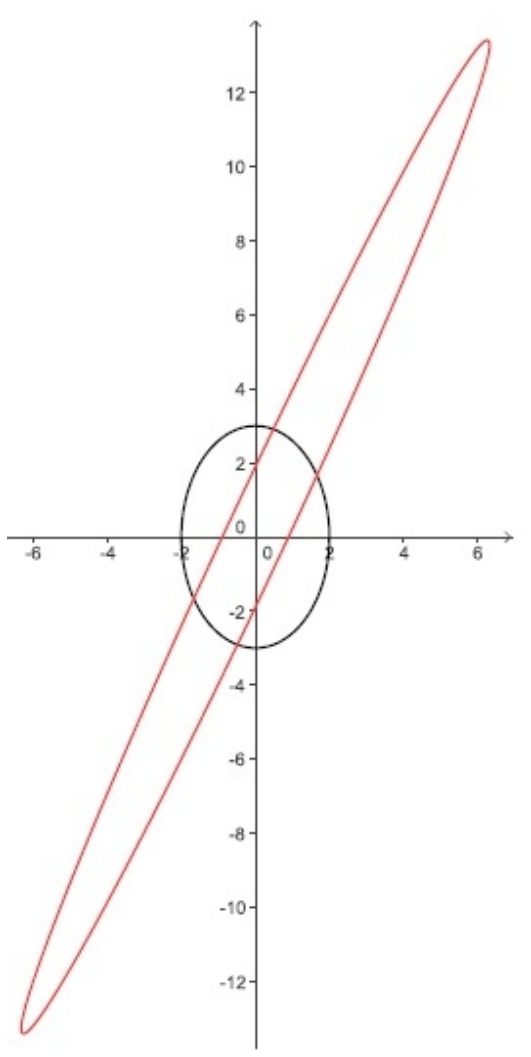

Figura 2: Imagem da elipse de equação $\frac{x^{2}}{4}+\frac{y^{2}}{9}=1$ segundo o operador $T_{M}(x, y)=(x+2 y, 3 x+4 y)$.

\section{$2.4 \epsilon$ é uma elipse qualquer}

Uma elipse $\epsilon$ qualquer do plano terá equação

$$
\frac{\widetilde{x}^{2}}{p^{2}}+\frac{\widetilde{\tilde{y}}^{2}}{q^{2}}=1
$$


no sistema $\widetilde{\widetilde{x}} O^{\prime} \widetilde{\widetilde{y}}$ que é oriundo de uma rotação de ângulo $\theta$ no sentido anti-horário do sistema $\widetilde{x} O^{\prime} \widetilde{y}$ onde este último é a translação de

$$
O^{\prime}=\left(\begin{array}{l}
x_{0} \\
y_{0}
\end{array}\right)
$$

do sistema $x O y$. Se considerar, por exemplo, uma elipse de equação

$$
\frac{\left(x-x_{0}\right)^{2}}{p^{2}}+\frac{\left(y-y_{0}\right)^{2}}{q^{2}}=1
$$

então os sistemas $\widetilde{\widetilde{x}} O^{\prime} \widetilde{y}$ e $\widetilde{x} O^{\prime} \widetilde{y}$ coincidem ao passo que se considera-se uma elipse de equação

$$
\frac{x^{2}}{p^{2}}+\frac{y^{2}}{q^{2}}=1
$$

então os sistemas $\widetilde{\widetilde{x}} O^{\prime} \widetilde{\tilde{y}}, \widetilde{x} O^{\prime} \widetilde{y}$ e $x O y$ coincidem. Desta forma, tem-se que

$$
\left(\begin{array}{c}
\tilde{x} \\
\widetilde{y}
\end{array}\right)=R_{\theta}\left(\begin{array}{c}
\widetilde{\widetilde{x}} \\
\widetilde{y}
\end{array}\right)
$$

e

$$
\left(\begin{array}{l}
x \\
y
\end{array}\right)=\left(\begin{array}{l}
\tilde{x} \\
\widetilde{y}
\end{array}\right)+\left(\begin{array}{l}
x_{0} \\
y_{0}
\end{array}\right)
$$

onde

$$
R_{\theta}=\left(\begin{array}{cc}
\cos \theta & -\sin \theta \\
\sin \theta & \cos \theta
\end{array}\right) .
$$

Seja $(x, y)$ um ponto arbitrário da elipse $\epsilon$. Mostra-se que a imagem $(u, v)$ de $(x, y)$ segundo o operador

$$
T_{M}\left(\begin{array}{l}
x \\
y
\end{array}\right)=M\left(\begin{array}{l}
x \\
y
\end{array}\right)=\left(\begin{array}{ll}
a & b \\
c & d
\end{array}\right)\left(\begin{array}{l}
x \\
y
\end{array}\right)
$$

representa as coordenadas de uma elipse ou suas degenerações. Com efeito, $(u, v)$ é tal que:

$$
\begin{aligned}
\left(\begin{array}{l}
u \\
v
\end{array}\right) & =M\left(\begin{array}{l}
x \\
y
\end{array}\right) \\
& =M\left(\left(\begin{array}{c}
\widetilde{x} \\
\widetilde{y}
\end{array}\right)+\left(\begin{array}{l}
x_{0} \\
y_{0}
\end{array}\right)\right) \\
& =M\left(R_{\theta}\left(\begin{array}{c}
\widetilde{\widetilde{x}} \\
\widetilde{\widetilde{y}}
\end{array}\right)+\left(\begin{array}{l}
x_{0} \\
y_{0}
\end{array}\right)\right) \\
& =M R_{\theta}\left(\begin{array}{c}
\widetilde{\widetilde{x}} \\
\widetilde{\widetilde{y}}
\end{array}\right)+M\left(\begin{array}{l}
x_{0} \\
y_{0}
\end{array}\right) .
\end{aligned}
$$

Escrevendo $M R_{\theta}=\widetilde{M}$ e tomando

$$
M\left(\begin{array}{l}
x_{0} \\
y_{0}
\end{array}\right)=\left(\begin{array}{l}
u_{0} \\
v_{0}
\end{array}\right)
$$

conclui-se que

$$
\left(\begin{array}{c}
u \\
v
\end{array}\right)=\widetilde{M}\left(\begin{array}{c}
\widetilde{\widetilde{x}} \\
\widetilde{\widetilde{y}}
\end{array}\right)+\left(\begin{array}{c}
u_{0} \\
v_{0}
\end{array}\right)
$$

$$
\left(\begin{array}{c}
u-u_{0} \\
v-v_{0}
\end{array}\right)=\widetilde{M}\left(\begin{array}{c}
\widetilde{\widetilde{x}} \\
\widetilde{\widetilde{y}}
\end{array}\right) .
$$

Tomando $u-u_{0}=\widetilde{u}, v-v_{0}=\widetilde{v}$ e substituindo em (6) tem-se

$$
\left(\begin{array}{c}
\widetilde{u} \\
\widetilde{v}
\end{array}\right)=\tilde{M}\left(\begin{array}{c}
\widetilde{\widetilde{x}} \\
\widetilde{\widetilde{y}}
\end{array}\right)
$$

As coordenadas $(\widetilde{u}, \widetilde{v})$ podem ser encaradas como $(u, v)$ a menos de uma translação. Da equação (7) concluise que $(\widetilde{u}, \widetilde{v})$ representa as coordenadas de uma elipse ou suas degenerações já que se trata da imagem da elipse

$$
\frac{\widetilde{\widetilde{x}}^{2}}{p^{2}}+\frac{\widetilde{\widetilde{y}}^{2}}{q^{2}}=1
$$

no referencial $\widetilde{\widetilde{x}} O^{\prime} \widetilde{\tilde{y}}$ pela aplicação

$$
T_{M}\left(\begin{array}{c}
\widetilde{\widetilde{x}} \\
\widetilde{\widetilde{y}}
\end{array}\right)=\widetilde{M}\left(\begin{array}{c}
\widetilde{\widetilde{x}} \\
\widetilde{\widetilde{y}}
\end{array}\right)+\left(\begin{array}{c}
u_{0} \\
v_{0}
\end{array}\right) .
$$

Se a matriz $M$ for tal que $\operatorname{det} M=0$ tem-se como imagem de $\epsilon$ uma degeneração da elipse, pois

$$
\operatorname{det} \widetilde{M}=\operatorname{det}\left(M R_{\theta}\right)=\operatorname{det} M \cdot \operatorname{det} R_{\theta}=\operatorname{det} M=0 .
$$

Se a matriz $M$ for tal que $\operatorname{det} M \neq 0$ tem-se como imagem de $\epsilon$ uma elipse, pois $\operatorname{det} \widetilde{M}=\operatorname{det} M \neq 0$. Neste último caso, pela subseção anterior, $T_{M}(\epsilon)$ tem por equação

$$
\lambda_{1}\left(\widetilde{\widetilde{u}}-\widetilde{\widetilde{u_{0}}}\right)^{2}+\lambda_{2}\left(\widetilde{\widetilde{v}}-\widetilde{\widetilde{v_{0}}}\right)^{2}=1,
$$

onde $\lambda_{1}$ e $\lambda_{2}$ são os autovalores da matriz

$$
S=\left(\widetilde{M}^{-1}\right)^{t}\left(\begin{array}{cc}
\frac{1}{p^{2}} & 0 \\
0 & \frac{1}{q^{2}}
\end{array}\right) \widetilde{M}^{-1}
$$

e $\widetilde{\widetilde{u}} \widetilde{v}$ é o referencial gerado pela base $\beta$ dos respectivos autovetores ortonormais desta e $\left(\widetilde{\tilde{u}_{0}}, \widetilde{\widetilde{v_{0}}}\right)$ são as coordenadas do vetor $\left(u_{0}, v_{0}\right)$ na base $\beta$. Com isso, acaba-se de demonstrar a proposição 4 .

Proposição 4. Seja $T_{M}: \mathbb{R}^{2} \rightarrow \mathbb{R}^{2}$ um operador linear invertível associado a matriz $M$ na base canônica. Se $\epsilon$ é uma elipse centrada em $P\left(x_{0}, y_{0}\right)$ de semi-eixos $p$ e q assentados nos eixos paralelos aos respectivos vetores $(\cos (\theta), \sin (\theta)) e$ $(-\sin (\theta), \cos (\theta))$ então $T_{M}(\epsilon)$ é uma elipse dada pela equação (8), onde $\lambda_{1}$ e $\lambda_{2}$ são os autovalores da matriz

$$
S=\left(M^{-1}\right)^{t} R_{\theta}\left(\begin{array}{cc}
\frac{1}{p^{2}} & 0 \\
0 & \frac{1}{q^{2}}
\end{array}\right) R_{(-\theta)} M^{-1},
$$

$\widetilde{\widetilde{u}} \widetilde{v}$ é o referencial gerado pela base $\beta$ dos respectivos autovetores ortonormais desta matriz e $\left(\widetilde{u_{0}}, \widetilde{v_{0}}\right)=\left[T_{M}\left(x_{0}, y_{0}\right)\right]_{\beta}$. 
Sendo $R_{\theta}$ a matriz de rotação de ângulo $\theta$ no sentido antihorário.

Exemplo 2. Considere a elipse $\epsilon$ dada parametricamente por

$u(t)=\left(\begin{array}{cc}\cos \left(60^{\circ}\right) & -\sin \left(60^{\circ}\right) \\ \sin \left(60^{\circ}\right) & \cos \left(60^{\circ}\right)\end{array}\right)\left(\begin{array}{c}4 \cos (t) \\ 2 \sin (t)\end{array}\right)+\left(\begin{array}{l}1 \\ 2\end{array}\right)$

onde $t \in[0,2 \pi]$ segundo a transformação

$$
T_{M}\left(\begin{array}{l}
x \\
y
\end{array}\right)=\left(\begin{array}{ll}
1 & 2 \\
3 & 4
\end{array}\right)\left(\begin{array}{l}
x \\
y
\end{array}\right)
$$

Note que como $\operatorname{det} M \neq 0, T_{M}(\epsilon)$ é uma elipse. A elipse $\epsilon$ tem semi-eixos $p=4$ e $q=2$ assentados nos eixos paralelos aos respectivos vetores $\left(\cos 60^{\circ}, \sin 60^{\circ}\right) e\left(-\sin 60^{\circ}, \cos 60^{\circ}\right) e$ está centrada centrada em $(1,2)$. Logo, $T_{M}(\epsilon)$ terá equação

$$
0.0021(\widetilde{\widetilde{u}}-12.0829)^{2}+1.8553(\widetilde{\widetilde{v}}-0.0676)^{2}=1 .
$$

A imagem da elipse resultante é apresentada na figura 3.

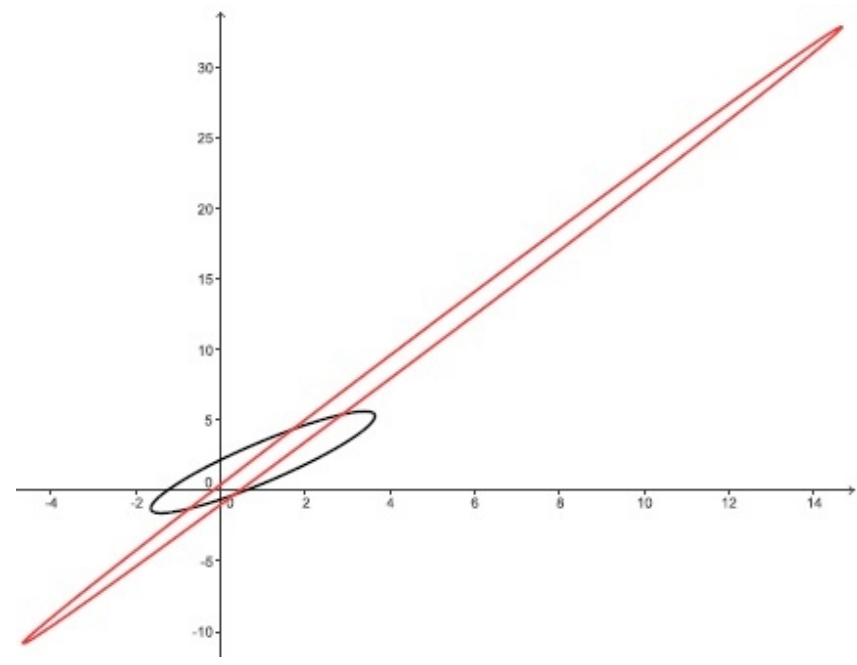

Figura 3: Imagem da elipse dada pela equação paramétrica $u(t)$ segundo o operador $T_{M}(x, y)=(x+2 y, 3 x+4 y)$.

O referencial $\widetilde{\widetilde{u}} \widetilde{v}$ é gerado base

$$
\begin{aligned}
& \left\{\left(\frac{2585}{\sqrt{M}},\right.\right. \\
& \left.\frac{-(6 \sqrt{3}-25) \sqrt{13860 \sqrt{3}+32261}+114 \sqrt{3}+2110}{\sqrt{M}}\right), \\
& \left(\frac{2585}{\sqrt{M}},\right. \\
& \left.\left.\frac{(6 \sqrt{3}-25) \sqrt{13860 \sqrt{3}+32261}+114 \sqrt{3}+2110}{\sqrt{M}}\right)\right\} .
\end{aligned}
$$

onde $M=-\sqrt{13860 \sqrt{3}+32261}(19620 \sqrt{3}-101396)$

$+962160 \sqrt{3}+22346626$.

\section{Deformação da hipérbole}

O estudo da hipérbole é análogo ao da elipse. Tem-se que se o operador

$$
T_{M}\left(\begin{array}{l}
x \\
y
\end{array}\right)=M\left(\begin{array}{l}
x \\
y
\end{array}\right)=\left(\begin{array}{ll}
a & b \\
c & d
\end{array}\right)\left(\begin{array}{l}
x \\
y
\end{array}\right),
$$

é tal que $\operatorname{det} M \neq 0$, a imagem de uma hipérbole da forma

$$
\frac{x^{2}}{p^{2}}-\frac{y^{2}}{q^{2}}=1
$$

será uma hipérbole. Se tomar uma hipérbole arbitrária (que estaria rotacionada e transladada em relação ao referencial $x O y$ ) mostra-se como em (14) que sua imagem segundo $T_{M}$ invertível também é uma hipérbole. Na verdade existe uma "dualidade" entre a elipse de equação (1) e a hipérbole de equação (9), ou seja, hipérboles vão em hipérboles e a mesma relação que existe entre a elipse original e a hipérbole associada será preservada na deformação pelo operador linear.

\section{Deformação da parábola}

Nesta seção, analisa-se a imagem de uma parábola segundo um operador linear no plano. Seja $T_{M}: \mathbb{R}^{2} \rightarrow \mathbb{R}^{2}$ um operador linear definido por

$$
T_{M}\left(\begin{array}{l}
x \\
y
\end{array}\right)=M\left(\begin{array}{l}
x \\
y
\end{array}\right)=\left(\begin{array}{ll}
a & b \\
c & d
\end{array}\right)\left(\begin{array}{l}
x \\
y
\end{array}\right),
$$

e uma parábola qualquer (que pode estar rotacionada e transladada em relação ao referencial $x O y$ ). Mostra-se que a imagem da parábola segundo $T_{M}$ é uma parábola ou suas degenerações (ponto, semi-reta ou mesmo uma reta). Nas subseções 2.1 a 2.4 estuda-se uma parábola $\sigma$ de equação

$$
y=l x^{2}
$$

onde serão discutidos todos os possíveis tipos de operadores no plano.

\section{1 $\sigma$ é uma parábola com vértice na origem e não rotacionada e $T_{M}$ é o operador nulo}

Neste caso, a imagem da parábola será o vetor nulo, já que $T_{M}(v)=0$ para todo $v \in \mathbb{R}^{2}$. Desta forma, a imagem da parábola é um ponto (parábola degenerada).

\section{2 $\sigma$ é uma parábola com vértice na origem e não rotacionada e $T_{M}$ é um operador não nulo onde $\operatorname{posto}(M)=1$}

Neste caso, tem-se $a d-b c=0, \log$ os vetores $\left(\begin{array}{l}a \\ b\end{array}\right)$ e $\left(\begin{array}{l}c \\ d\end{array}\right)$ são linearmente dependentes. Supondo sem 
perda de generalidade que $\left(\begin{array}{l}c \\ d\end{array}\right)=k\left(\begin{array}{l}a \\ b\end{array}\right)$, pode-se escrever

$$
T_{M}\left(\begin{array}{l}
x \\
y
\end{array}\right)=\left(\begin{array}{cc}
a & b \\
k a & k b
\end{array}\right)\left(\begin{array}{l}
x \\
y
\end{array}\right) .
$$

Tomando a representação paramétrica da parábola temse

$$
\left(\begin{array}{l}
x \\
y
\end{array}\right)=\left(\begin{array}{c}
t \\
l t^{2}
\end{array}\right), t \in \mathbb{R}
$$

$\log 0$

$$
\begin{array}{r}
T_{M}\left(\begin{array}{c}
x \\
y
\end{array}\right)=T_{M}\left(\begin{array}{c}
t \\
l t^{2}
\end{array}\right)=\left(\begin{array}{cc}
a & b \\
k a & k b
\end{array}\right)\left(\begin{array}{c}
t \\
l t^{2}
\end{array}\right) \\
=\left(a t+b l t^{2}\right)\left(\begin{array}{l}
1 \\
k
\end{array}\right)=f(t)\left(\begin{array}{l}
1 \\
k
\end{array}\right)
\end{array}
$$

onde $f(t)=a t+b l t^{2}$. Desta forma, $f(t)\left(\begin{array}{l}1 \\ k\end{array}\right)$ representará a equação paramétrica de uma semi-reta ou de uma reta conforme $b \neq 0$ ou $b=0$, respectivamente.

\section{1ํ Caso: $b \neq 0$}

Neste caso, a função $f(t)$ tem máximo ou mínimo dependendo do sinal de $b l$, portanto $T_{M}(\sigma)$ representa uma semi-reta cujo vetor diretor é $\left(\begin{array}{l}1 \\ k\end{array}\right)$ de vértice em $V=\left(\begin{array}{c}\frac{-a^{2}}{4 l b} \\ \frac{-k a^{2}}{4 l b}\end{array}\right)$. Esta semi-reta estará "acima" ou "abaixo" de $V$ conforme a função $k f(t)$ tenha máximo ou mínimo respectivamente.

\section{$2^{\underline{o}}$ Caso: $b=0$}

Neste caso, a função $f(t)$ é linear, portanto ilimitada superiormente e inferiormente. Logo, segue que $T_{M}(\sigma)$ representa uma reta cujo vetor diretor é $\left(\begin{array}{l}1 \\ k\end{array}\right)$. Com isso, tem-se a proposição 5 .

Proposição 5. Seja $M$ uma matriz $2 \times 2$ de posto 1 , ou seja, M é de uma das formas:

$$
\begin{gathered}
\left(\begin{array}{cc}
a & b \\
k a & k b
\end{array}\right) \text { ou }\left(\begin{array}{cc}
k a & k b \\
a & b
\end{array}\right), \\
(a, b) \neq(0,0) .
\end{gathered}
$$

Seja $T_{M}: \mathbb{R}^{2} \rightarrow \mathbb{R}^{2}$ um operador associado a matriz $M n a$ base canônica. Se $\sigma$ é uma parábola de equação $y=l x^{2}$ tem-se:

\section{(i) Caso $b \neq 0$.}

$T_{M}(\sigma)$ é uma semi-reta cujo vetor diretor é $(1, k)$ ou $(k, 1)$, conforme $M$ tenha respectivamente a $1^{\underline{a}}$ ou $2^{\underline{a}}$ representação dada anteriormente. Além disso, tem-se:

a) Se $(1, k)$ é o vetor diretor, então a semi-reta da imagem tem vértice dado por $V=\left(\frac{-a^{2}}{4 l b}, \frac{-k a^{2}}{4 l b}\right)$ e está "acima" ou "abaixo" deste, conforme $\mathrm{kbl}>0$ ou $\mathrm{kbl}<0$ respectivamente.

b) Se $(k, 1)$ é o vetor diretor, então a semi-reta da imagem tem vértice dado por $V=\left(\frac{-k a^{2}}{4 l b}, \frac{-a^{2}}{4 l b}\right)$ e estará "acima" ou "abaixo" deste, conforme bl $>0$ ou bl $<0$ respectivamente.

(ii) Caso $b=0$.

$T_{M}(\sigma)$ é a reta pela origem cujo vetor diretor é $(1, k)$ ou $(k, 1)$ conforme $M$ tenha respectivamente a $1^{\underline{a}}$ ou $2^{\underline{a}}$ representação dada anteriormente.

Exemplo 3. Considere a parábola $y=x^{2}$ segundo a transformação

$$
T_{M}\left(\begin{array}{l}
x \\
y
\end{array}\right)=\left(\begin{array}{cc}
5 & -3 \\
-10 & 6
\end{array}\right)\left(\begin{array}{l}
x \\
y
\end{array}\right) .
$$

Escreve-se a parábola na forma

$$
u(t)=\left(\begin{array}{c}
t \\
t^{2}
\end{array}\right)
$$

onde $t \in[-2,2]$. A imagem da parábola pode ser visualizada na figura 4.

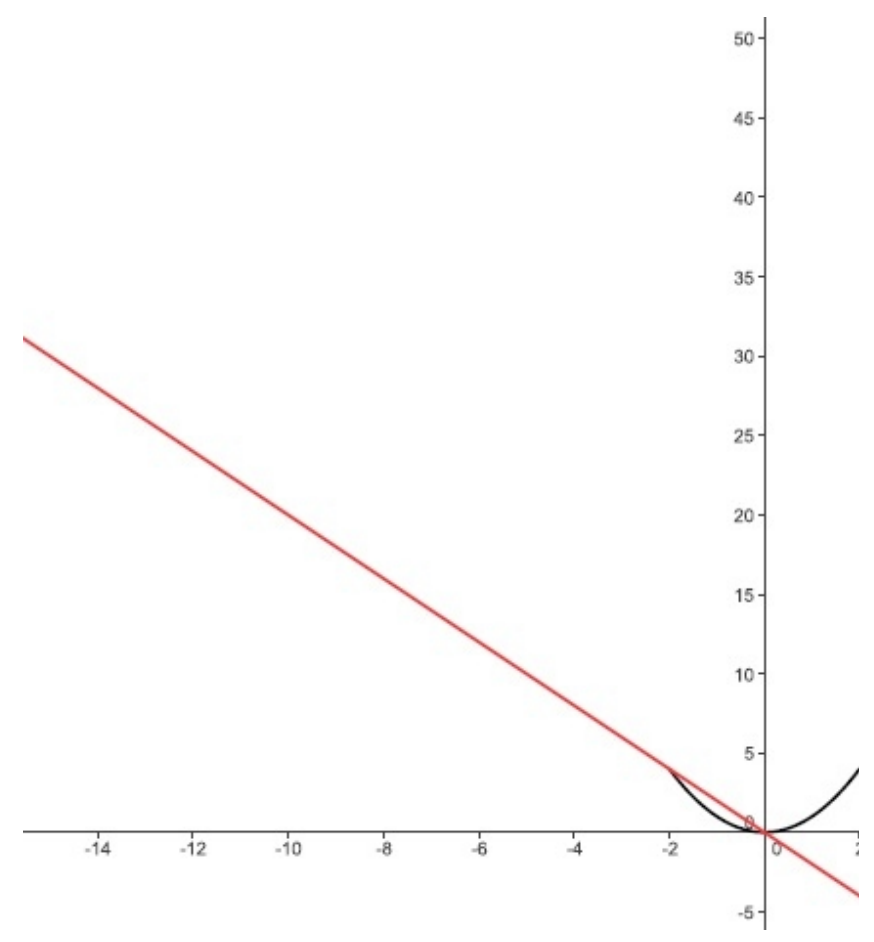

Figura 4: Imagem da parábola de equação $y=x^{2}$ segundo o operador $T_{M}(x, y)=(5 x-3 y,-10 x+6 y)$. 
Note que, neste caso, a imagem da parábola é uma semireta, pois $M$ tem posto 1 e $b \neq 0$. Esta semi-reta tem como vetor diretor $(1,-2)$ e está "acima" do vértice $V=\left(\begin{array}{c}\frac{25}{12} \\ -\frac{25}{6}\end{array}\right)$.

\section{3 $\sigma$ é uma parábola com vértice na origem e não rotacionada e $T_{M}$ é um operador invertível}

Mostra-se que a imagem de $\sigma$ segundo o operador $T_{M}$ é uma parábola cuja equação determina-se. Reescrevendo a equação de $\sigma$ em forma matricial tem-se:

$$
\left(\begin{array}{ll}
x & y
\end{array}\right)\left(\begin{array}{ll}
l & 0 \\
0 & 0
\end{array}\right)\left(\begin{array}{l}
x \\
y
\end{array}\right)+\left(\begin{array}{ll}
0 & -1
\end{array}\right)\left(\begin{array}{l}
x \\
y
\end{array}\right)=0 .
$$

Admitindo que $T_{M}(x, y)=(u, v)$ e que $T_{M}$ é invertível tem-se que

$$
\left(\begin{array}{l}
x \\
y
\end{array}\right)=M^{-1}\left(\begin{array}{l}
u \\
v
\end{array}\right) .
$$

Substituindo este resultado em (10) obtêm-se

$$
\begin{array}{r}
\left(\begin{array}{ll}
u & v
\end{array}\right)\left(M^{-1}\right)^{t}\left(\begin{array}{ll}
l & 0 \\
0 & 0
\end{array}\right) M^{-1}\left(\begin{array}{l}
u \\
v
\end{array}\right) \\
+\left(\begin{array}{ll}
0 & -1
\end{array}\right) M^{-1}\left(\begin{array}{l}
u \\
v
\end{array}\right)=0 \\
\left(\begin{array}{ll}
u & v
\end{array}\right) S\left(\begin{array}{l}
u \\
v
\end{array}\right)+N\left(\begin{array}{l}
u \\
v
\end{array}\right)=0,
\end{array}
$$

onde

$$
S=\left(M^{-1}\right)^{t}\left(\begin{array}{ll}
l & 0 \\
0 & 0
\end{array}\right) M^{-1}
$$

e

$$
N=\left(\begin{array}{ll}
0 & -1
\end{array}\right) M^{-1}
$$

Note que $S$ é simétrica e então existe $P$ ortonormal e $D$ diagonal tal que $S=P D P^{t}$. Desta forma, (11) pode ser reescrita como

$$
\left(\begin{array}{cc}
u & v
\end{array}\right) P\left(\begin{array}{cc}
\lambda_{1} & 0 \\
0 & \lambda_{2}
\end{array}\right) P^{t}\left(\begin{array}{l}
u \\
v
\end{array}\right)+N P P^{t}\left(\begin{array}{l}
u \\
v
\end{array}\right)=0 .
$$

Tomando

$$
\left(\begin{array}{c}
u \\
v
\end{array}\right)=P\left(\begin{array}{c}
\widetilde{u} \\
\widetilde{v}
\end{array}\right) \quad \text { e } \quad D=\left(\begin{array}{cc}
\lambda_{1} & 0 \\
0 & \lambda_{2}
\end{array}\right),
$$

segue que

$$
\left(\begin{array}{cc}
\widetilde{u} & \widetilde{v}
\end{array}\right)\left(\begin{array}{cc}
\lambda_{1} & 0 \\
0 & \lambda_{2}
\end{array}\right)\left(\begin{array}{c}
\widetilde{u} \\
\widetilde{v}
\end{array}\right)+N P\left(\begin{array}{c}
\widetilde{u} \\
\widetilde{v}
\end{array}\right)=0 .
$$

Escrevendo $N P=\left(\begin{array}{cc}\alpha & \beta\end{array}\right)$, obtém-se

$$
\lambda_{1}(\widetilde{u})^{2}+\lambda_{2}(\widetilde{v})^{2}+\alpha \widetilde{u}+\beta \widetilde{v}=0 .
$$

A matriz $S$ é dada por $S=\left(\begin{array}{ll}\widetilde{a} & \widetilde{b} \\ \widetilde{b} & \widetilde{c}\end{array}\right)$, onde

$$
\begin{aligned}
\widetilde{a} & =\frac{d^{2} l}{(\operatorname{det} M)^{2}} \\
\widetilde{b} & =-\frac{b d l}{(\operatorname{det} M)^{2}} \\
\widetilde{c} & =\frac{b^{2} l}{(\operatorname{det} M)^{2}} .
\end{aligned}
$$

Os autovalores e os respectivos autovetores associados de $S$, após serem normalizados, são

$$
\begin{aligned}
& \lambda_{1}=\frac{\left(d^{2}+b^{2}\right) l}{(\operatorname{det} M)^{2}} \\
& \lambda_{2}=0 \\
& u_{1}=\left(\frac{d}{\sqrt{d^{2}+b^{2}}},-\frac{b}{\sqrt{d^{2}+b^{2}}}\right) \\
& u_{2}=\left(\frac{b}{\sqrt{d^{2}+b^{2}}}, \frac{d}{\sqrt{d^{2}+b^{2}}}\right) .
\end{aligned}
$$

Logo, a equação (12) pode ser assim reescrita

$$
\lambda_{1}(\widetilde{u})^{2}+\alpha \widetilde{u}+\beta \widetilde{v}=0,
$$

donde conclui-se que $T_{M}(\sigma)$ é uma parábola. Com isso, acaba-se de demonstrar a proposição 6 .

Proposição 6. Seja $T_{M}: \mathbb{R}^{2} \rightarrow \mathbb{R}^{2}$ um operador linear invertível associado a matriz $M$ na base canônica. Se $\sigma$ é uma parábola de equação

$$
y=l x^{2}
$$

então $T_{M}(\sigma)$ é uma parábola dada pela equação (13), onde $\lambda_{1}$ é o autovalor não nulo da matriz simétrica

$$
\begin{aligned}
& S=\left(M^{-1}\right)^{t}\left(\begin{array}{ll}
l & 0 \\
0 & 0
\end{array}\right) M^{-1}, \\
& \left(\begin{array}{ll}
\alpha & \beta
\end{array}\right)=\left(\begin{array}{ll}
0 & -1
\end{array}\right) M^{-1} P
\end{aligned}
$$

e $\widetilde{u} \widetilde{v}$ é o referencial gerado pelos autovetores ortonormais de $S$.

Exemplo 4. Considere a parábola $y=x^{2}$ segundo a transformação

$$
T_{M}\left(\begin{array}{l}
x \\
y
\end{array}\right)=\left(\begin{array}{ll}
1 & 2 \\
3 & 4
\end{array}\right)\left(\begin{array}{l}
x \\
y
\end{array}\right)
$$

Para isso escreve-se a parábola parametricamente

$$
u(t)=\left(\begin{array}{c}
t \\
t^{2}
\end{array}\right)
$$

onde $t \in[-2,2]$. A imagem da parábola pode ser visualizada na figura 5 . 


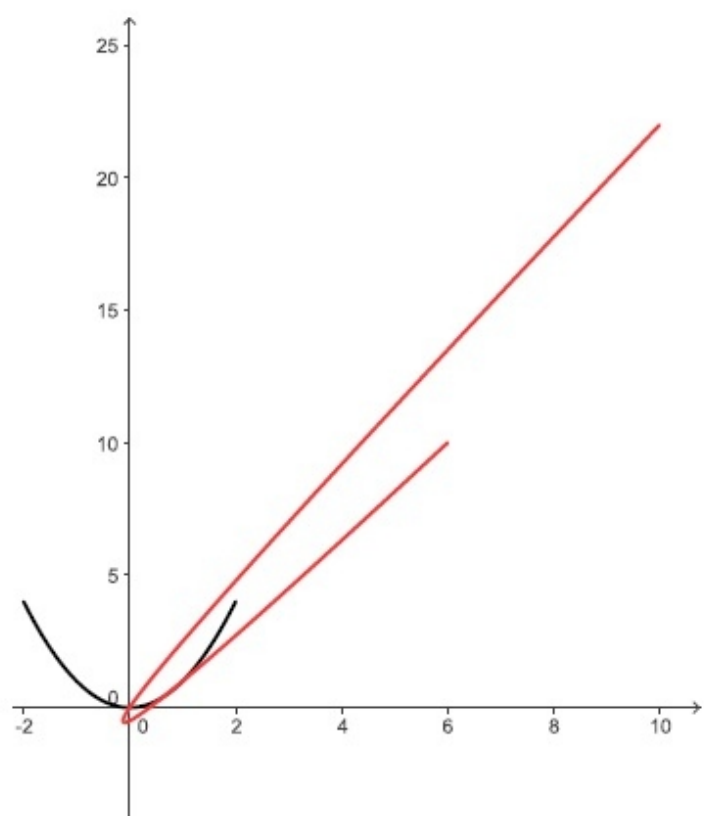

Figura 5: Imagem da parábola de equação $y=x^{2}$ segundo o operador $T_{M}(x, y)=(x+2 y, 3 x+4 y)$.

Neste caso, como det $M \neq 0$, a imagem da parábola $y=x^{2}$ é uma parábola, e esta terá equação

$$
5(\widetilde{u})^{2}+\frac{7}{2 \sqrt{5}} \widetilde{u}-\frac{1}{2 \sqrt{5}} \widetilde{v}=0,
$$

onde $\widetilde{u} \widetilde{v}$ é o referencial gerado pela base

$$
\left\{\left(-\frac{2}{\sqrt{5}}, \frac{1}{\sqrt{5}}\right),\left(\frac{1}{\sqrt{5}}, \frac{2}{\sqrt{5}}\right)\right\},
$$

\section{4 $\sigma$ é uma parábola qualquer.}

Uma parábola $\sigma$ qualquer do plano terá equação

$$
\widetilde{\widetilde{y}}=l(\widetilde{\widetilde{x}})^{2}
$$

no sistema $\widetilde{\widetilde{x}} O^{\prime} \widetilde{\widetilde{y}}$ que é oriundo de uma rotação de ângulo $\theta$ no sentido anti-horário do sistema $\widetilde{x} O^{\prime} \widetilde{y}$ onde este último é a translação de

$$
\mathrm{O}^{\prime}=\left(\begin{array}{l}
x_{0} \\
y_{0}
\end{array}\right)
$$

do sistema $x O y$. Da subseção 2.4, tem-se que

$$
\left(\begin{array}{c}
\tilde{u} \\
\widetilde{v}
\end{array}\right)=\tilde{M}\left(\begin{array}{c}
\widetilde{\widetilde{x}} \\
\widetilde{\widetilde{y}}^{\prime}
\end{array}\right)
$$

onde

$$
\left(\begin{array}{l}
\widetilde{u} \\
\widetilde{v}
\end{array}\right)=\left(\begin{array}{l}
u \\
v
\end{array}\right)-\left(\begin{array}{l}
u_{0} \\
v_{0}
\end{array}\right)
$$

$$
\left(\begin{array}{l}
u_{0} \\
v_{0}
\end{array}\right)=M\left(\begin{array}{l}
x_{0} \\
y_{0}
\end{array}\right)
$$

e $M R_{\theta}=\tilde{M}$. Da equação (14) conclui-se que

$$
\left(\begin{array}{c}
\widetilde{u} \\
\widetilde{v}
\end{array}\right)
$$

representa as coordenadas de uma parábola ou suas degenerações, já que se trata da imagem da parábola $\widetilde{\widetilde{y}}=l(\widetilde{\widetilde{x}})^{2}$ no referencial $\widetilde{\widetilde{x}} O^{\prime} \widetilde{\widetilde{y}}$ pela aplicação

$$
T_{\tilde{M}}\left(\begin{array}{c}
\widetilde{\widetilde{x}} \\
\widetilde{\widetilde{y}}
\end{array}\right)=\widetilde{M}\left(\begin{array}{c}
\widetilde{\widetilde{x}} \\
\widetilde{\widetilde{y}}
\end{array}\right)+\left(\begin{array}{l}
u_{0} \\
v_{0}
\end{array}\right) .
$$

Se a matriz $M$ for tal que $\operatorname{det} M=0$ tem-se como imagem de $\sigma$ uma degeneração da parábola, pois $\operatorname{det} \widetilde{M}=\operatorname{det} M=0$. Se a matriz $M$ for tal que $\operatorname{det} M \neq 0$ tem-se como imagem de $\sigma$ uma parábola, pois

$$
\operatorname{det} \tilde{M}=\operatorname{det} M \neq 0 .
$$

Neste último caso, pela subseção anterior, $T_{M}(\sigma)$ satisfaz a equação

$$
\lambda_{1}\left(\widetilde{u}-\widetilde{u_{0}}\right)^{2}+\alpha\left(\widetilde{u}-\widetilde{u_{0}}\right)+\beta\left(\widetilde{v}-\widetilde{v_{0}}\right)=0,
$$

onde $\lambda_{1}$ é o autovalor não nulo da matriz

$$
S=\left(\widetilde{M}^{-1}\right)^{t}\left(\begin{array}{ll}
l & 0 \\
0 & 0
\end{array}\right)(\tilde{M})^{-1}
$$

$\widetilde{u} \widetilde{v}$ é o referencial gerado pelos autovetores ortonormais de $S,\left(\widetilde{u_{0}}, \widetilde{v_{0}}\right)$ são as coordenadas do vetor $\left(u_{0}, v_{0}\right)$ na base de autovetores de $S$ e

$$
\left(\begin{array}{ll}
\alpha & \beta
\end{array}\right)=\left(\begin{array}{ll}
0 & -1
\end{array}\right)(\tilde{M})^{-1} P
$$

onde $P$ é a matriz cujas colunas são os autovetores ortonormais de $\mathrm{S}$. Com isso, acaba-se de demonstrar a proposição 7 .

Proposição 7. Seja $T_{M}: \mathbb{R}^{2} \rightarrow \mathbb{R}^{2}$ um operador linear invertível associado a matriz $M$ na base canônica. Se $\sigma$ é uma parábola de equação

$$
\widetilde{\widetilde{y}}=l(\widetilde{\widetilde{x}})^{2}
$$

no sistema $\widetilde{\widetilde{x}} \mathrm{O}^{\prime} \widetilde{\widetilde{y}}$, o qual é oriundo de uma rotação de ângulo $\theta$ no sentido anti-horário do sistema $\widetilde{x} O^{\prime} \widetilde{y}$, que por sua vez é a translação de

$$
O^{\prime}=\left(\begin{array}{l}
x_{0} \\
y_{0}
\end{array}\right)
$$

do sistema $x \mathrm{Oy}$. Então, $T_{M}(\sigma)$ é uma parábola dada pela equação (15), onde $\lambda_{1}$ é o autovalor não nulo da matriz

$$
S=\left(M^{-1}\right)^{t} R_{\theta}\left(\begin{array}{ll}
l & 0 \\
0 & 0
\end{array}\right) R_{(-\theta)} M^{-1}
$$


os coeficientes $\alpha$ e $\beta$ são dados por

$$
\left(\begin{array}{ll}
\alpha & \beta
\end{array}\right)=\left(\begin{array}{ll}
0 & -1
\end{array}\right) R_{(-\theta)} M^{-1} P,
$$

$\widetilde{\widetilde{u}} \widetilde{\widetilde{v}}$ é referencial gerado pela base $\widetilde{\beta}$ de autovetores da matriz $S, P=[I]_{\text {canônica }}^{\widetilde{\beta}} e\left(\widetilde{u_{0}}, \widetilde{v_{0}}\right)=\left[T_{M}\left(x_{0}, y_{0}\right)\right]_{\widetilde{\beta}}$. Sendo $R_{\theta} a$ matriz de rotação de ângulo $\theta$ no sentido anti-horário.

Exemplo 5. Considere a parábola dada parametricamente por

$$
u(t)=\left(\begin{array}{cc}
\cos 60^{\circ} & -\sin 60^{\circ} \\
\sin 60^{\circ} & \cos 60^{\circ}
\end{array}\right)\left(\begin{array}{c}
t \\
t^{2}
\end{array}\right)+\left(\begin{array}{l}
2 \\
3
\end{array}\right),
$$

onde $t \in[-2,8]$, segundo a transformação

$$
T_{M}\left(\begin{array}{l}
x \\
y
\end{array}\right)=\left(\begin{array}{ll}
1 & 2 \\
3 & 4
\end{array}\right)\left(\begin{array}{l}
x \\
y
\end{array}\right) .
$$

Note que, neste caso, a imagem da parábola é uma parábola, pois $\operatorname{det} M \neq 0$. A imagem da parábola pode ser visualizada na figura 6.

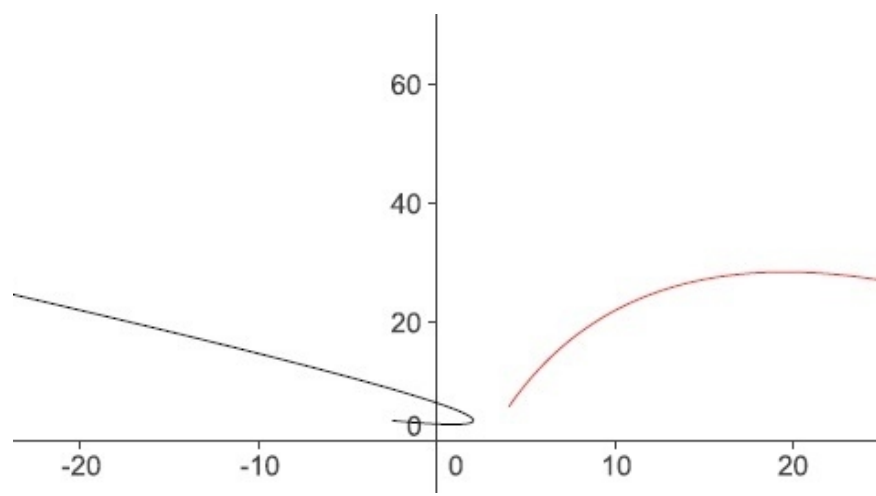

Figura 6: Imagem da parábola de equação paramétrica $u(t)$ segundo o operador $T_{M}(x, y)=(x+2 y, 3 x+4 y)$.

Esta parábola tem por equação

$$
\begin{array}{r}
\left(\frac{25-14 \sqrt{3}}{8}\right)\left(\widetilde{u}-\frac{32 \sqrt{3}+105}{\sqrt{2} \sqrt{67-2 \sqrt{3}}}\right)^{2} \\
-\left(\frac{11(2 \sqrt{3}+1)}{2 \sqrt{2} \sqrt{67-2 \sqrt{3}}}\right)\left(\widetilde{u}-\frac{32 \sqrt{3}+105}{\sqrt{2} \sqrt{67-2 \sqrt{3}}}\right) \\
-\left(\frac{2(\sqrt{3}+2)}{\sqrt{2} \sqrt{2 \sqrt{3}+7}}\right)\left(\widetilde{v}+\frac{32 \sqrt{3}+5}{\sqrt{2} \sqrt{2 \sqrt{3}+7}}\right)=0
\end{array}
$$

onde $\widetilde{u} \widetilde{v}$ é o referencial gerado pela base

$$
\begin{array}{r}
\left\{\left(\frac{11}{\sqrt{134-4 \sqrt{3}}}, \frac{2 \sqrt{3}-1}{\sqrt{134-4 \sqrt{3}}}\right),\right. \\
\left.\left(\frac{1}{\sqrt{4 \sqrt{3}+14}}, \frac{-2 \sqrt{3}-1}{\sqrt{4 \sqrt{3}+14}}\right)\right\} .
\end{array}
$$

\section{Conclusões}

No presente estudo, analisa-se as deformações de cônicas por transformações lineares. Tais deformações foram obtidas usando-se a forma paramétrica de uma cônica para encontrar a equação de sua imagem quando o operador linear está associado a uma matriz de posto incompleto. No caso em que o operador linear é invertível, estuda-se os autovalores da matriz simétrica associada à forma quadrática da cônica original e a partir daí encontra-se a equação de sua imagem no referencial gerado pelos autovetores desta matriz. De forma semelhante, de acordo com Tavares (2008), analisa-se as deformações de quádricas por transformações lineares no espaço.

\section{Referências}

Boldrini, J. L., Costa, S., Figueiredo, V., Wetzler, H. (1986). Álgebra Linear. ampl. e rev. São Paulo: HARBRA.

Coelho, F. U., Lourenço, M. L. (2001). Curso de Álgebra Linear, Um Vol. 34. Edusp.

Howard, A., Rorres, C. (2001). Álgebra Linear com aplicações, vol 8.

Lang, S. (2004). Álgebra Linear. Ed., Editora Ciência Moderna.

Lima, E. L. (1996). Álgebra Linear, 2a. ediçao. IMPA, Rio de Janeiro.

Tavares, F. P. (2008). Deformações de cônicas e quádricas por operadores lineares. Unicamp. 